\title{
BMJ An exploratory study on the open consequences and contextual factors of intimate partner violence among immigrant and Canadian-born women
}

\author{
Janice Du Mont, ${ }^{1,2}$ Tonia Forte ${ }^{1}$
}

To cite: Du Mont J, Forte T. An exploratory study on the consequences and contextual factors of intimate partner violence among immigrant and Canadian-born women. BMJ Open 2012;2:e001728. doi:10.1136/bmjopen-2012001728

- Prepublication history for this paper are available online. To view these files please visit the journal online (http://dx.doi.org/10.1136/ bmjopen-2012-001728).

Received 25 June 2012 Accepted 27 September 2012

This final article is available for use under the terms of the Creative Commons Attribution Non-Commercial 2.0 Licence; see http://bmjopen.bmj.com

${ }^{1}$ Women's College Research Institute, Women's College Hospital, Toronto, Ontario, Canada

2Dalla Lana School of Public Health, University of Toronto, Ontario, Canada

\section{Correspondence to} Dr Janice Du Mont; janice.dumont@wchospital.ca

\section{ABSTRACT}

Objective: To compare immigrant and Canadian-born women on the physical and psychological consequences of intimate partner violence (IPV), as well as examine important sociodemographic, health and social support and network factors that may shape their experiences of abuse.

Method: National, population-based, cross-sectional survey conducted in 2009.

Participants: 6859 women reported contact with a current or former partner in the previous 5 years, of whom 1480 reported having experienced emotional, financial, physical and/or sexual IPV. Of these women, $218(15 \%)$ were immigrants and $1262(85 \%)$ were Canadian-born.

Results: Immigrant women were less likely than Canadian-born women to report having experienced emotional abuse ( $15.3 \%$ vs $18.2 \%, p=0.04)$ and physical and/or sexual violence $(5.1 \%$ vs $6.9 \%, p=0.04)$ from a current or former partner. There were no differences between immigrant and Canadian-born women in the physical and psychological consequences of physical and/ or sexual IPV. However, compared with Canadian-born women, immigrant women reported lower levels of trust towards their neighbours ( $50.7 \%$ vs $41.5 \%, \mathrm{p}=0.04$ ) and people they work or go to school with (38.6\% vs $27.5 \%$, $\mathrm{p}=0.02$ ), and were more likely to report having experienced discrimination based on ethnicity or culture ( $18.8 \%$ vs $6.8 \%, p<0.0001)$, race or skin colour $(\mathrm{p}=0.003)$ and language $(10.1 \%$ vs $3.2 \%, p<0.0001)$. Immigrant women were less likely than Canadian-born women to report activity limitations $(\mathrm{p}=0.01)$ and medication use for sleep problems ( $14.1 \%$ vs $20.6 \%, p=0.05)$ and depression ( $11.5 \%$ vs $17.6 \%, p=0.05)$.

Conclusions: Our exploratory study revealed no differences between immigrant and Canadian-born women in the physical and psychological consequences of IPV. Abused immigrant women's lower levels of trust for certain individuals and experiences of discrimination may have important implications for seeking help for IPV and underscores the need for IPV-related intervention and prevention services that are culturally sensitive and appropriate.

\section{INTRODUCTION}

The prevalence and physical and psychological effects of intimate partner violence

\section{ARTICLE SUMMARY}

Article focus

- To compare immigrant and Canadian-born women on the physical and psychological consequences of IPV.

- To investigate important sociodemographic, health and social support and network factors that may shape immigrant women's experiences of abuse.

\section{Key messages}

- The prevalence of emotional abuse and physical/ sexual violence was lower among immigrant compared with Canadian-born women.

- There were no differences between immigrant and Canadian-born women in the physical and psychological consequences of physical and/or sexual IPV.

- Abused immigrant women were more likely than Canadian-born women to report lower levels of trust and experiences of discrimination and this may have implications in seeking help for IPV.

Strengths and limitations of this study

- This exploratory study adds to the very limited body of research that has examined the physical and psychological consequences of IPV among immigrant compared with non-immigrant women.

- Future research investigating the physical and psychological consequences of IPV should include larger diverse samples of immigrant women.

(IPV), defined as physical, emotional, financial and/or sexual abuse perpetrated against the victim by an intimate partner are well documented among women in the general population. ${ }^{1-7}$ Little is known, however, about the extent and consequences of IPV among immigrant women. Although immigrant women are a heterogeneous group, factors related to their premigration and postmigration contexts, such as economic insecurity, family separation, social isolation, language barriers, availability of social 
supports, discrimination and gendered-migration policies $^{8-15}$ may increase their vulnerability to abuse. Such factors may also intensify the physical and psychological impacts of abuse, while adding to the challenges immigrant women may experience in seeking help and leaving abusive situations. . $^{15-18}$

Findings of the prevalence of IPV among immigrant women have been mixed. A few population-based studies have shown that the prevalence of any type of IPV is lower among immigrant women compared with non-immigrant women, ${ }^{19-21}$ with those recently settled (ie, $<10$ years) at significantly lower risk of abuse than longer-term immigrants. ${ }^{22}$ Another study showed that, while rates of physical abuse were similar, the prevalence of emotional abuse was significantly higher among recently immigrated women $(14.7 \%)$ compared with Canadian-born women $(8.7 \%){ }^{23}$ Smaller community-based studies with non-representative samples suggest that the prevalence of IPV among immigrant women is higher than prevalence rates reported from population-based surveys, ${ }^{24-26}$ and reach as high as $60 \%$ in some studies. ${ }^{14}$

We were able to locate only one study that compared immigrant and non-immigrant women with respect to the physical and mental health consequences of IPV. This California-based study found that, although foreignborn Latinas reported lower rates of physical, sexual and psychological IPV compared with US-born Latinas, injury rates were markedly higher among Latina women born outside of the USA. Two-fifths (39.3\%) of US-born women who were victims of lifetime physical violence reported injury, or indicated the need for or the use of medical care, compared with almost three-quarters $(73.1 \%)$ of immigrant women. ${ }^{19}$ No differences were noted in mental health outcomes between immigrant and US-born women who had experienced violence.

Given the lack of research examining the physical and psychological effects of IPV among immigrant women, the goal of this study was to compare immigrant and Canadian-born women in the physical and psychological consequences of abuse. As premigration and postmigration factors may shape immigrant women's experiences of abuse, we also sought to examine the differences between abused immigrant and Canadian-born women across several domains such as their health status and social supports and networks. This information may provide insights into how immigrant women's particular contexts might affect their experiences of abuse and better elucidate the health-related outcomes associated with being a victim of IPV.

\section{METHODS}

Statistics Canada's General Social Survey (GSS) is a national survey that in 2009 focused again on victimisation and perceptions and experiences of crime and safety. ${ }^{27}$ Canadians aged 15 years or older living in private households in 10 provinces were interviewed. Respondents were selected using a process of Random
Digit Dialing. Each province was divided into geographic areas or strata, and all phone numbers within each stratum had the same probability of being chosen. After a household was contacted, an individual 15 years of age or older within the household was selected at random to be interviewed. Data were collected using computerassisted telephone interviewing. Interviewers were trained in telephone interviewing techniques by Statistics Canada staff. Given the nature of the questions in the survey, they were also provided with crisis, sensitivity and personal preparedness training by a psychologist, in accordance with ethical and safety recommendations for research on IPV against women. ${ }^{28}$ Interviews were conducted between February and December 2009 and were administered in English or French. Respondents were assured that all information provided was strictly confidential. Of the 31510 households that were selected, 19422 usable responses were obtained, representing a response rate of $61.6 \%$.

\section{Definition of IPV}

Respondents were asked about their experiences of IPV by a current or former partner with whom they had had contact within the 5 years preceding the survey. This included respondents who were legally married, living common law, divorced or separated or in a same-sex relationship. Physical and sexual IPV were measured using the modified Conflict Tactics Scale.

- Physical violence was assessed by asking respondents whether a current or former partner had threatened to hit them; threw something at them; pushed, grabbed or shoved them; slapped them; kicked, bit or hit them with a fist; hit them with something that could hurt; beaten them; choked them or used or threatened to use a knife or gun on them.

- Sexual violence was assessed by asking respondents, 'Has your partner or former partner forced you into any unwanted sexual activity by threatening you, holding you down or hurting you in some way?'

- Emotional abuse was defined as having occurred if a respondent answered affirmatively to at least one of the following statements about her partner/former partner's behaviour: 'tried to limit your contact with family or friends, put you down or called you names to make you feel bad, was jealous and did not want you to talk to other men or women, harmed or threatened to harm someone close to you, demanded to know who you were with and where you were at all times and damaged or destroyed your possessions or property'.

- Financial abuse was measured by the question, 'Has your partner prevented you from knowing about or having access to the family income, even if you asked?'

In this study, any IPV was defined as one or more types of physical, sexual, emotional or financial abuse. The severity of IPV was conceptualised in terms of the number of different types of abuse experienced (ie, one 
type of physical, sexual, emotional or financial abuse vs two or more types).

\section{Immigration status}

The GSS assessed immigration status by asking respondents to indicate their country of birth. In this study, Canadian-born women included those women who indicated their country of birth to be Canada. Immigrant women included those women in the survey who indicated they were born outside of Canada.

\section{Sociodemographic characteristics}

Sociodemographic characteristics examined included age group (15-34, 35-54, 55 and older), marital status (married/common-law and widowed/separated/divorced/ single), highest level of education achieved (high-school graduate or less, more than high school), annual household income in Canadian dollars (0-\$19999; \$20 000$\$ 49$ 999; \$50 000 or more), presence of children younger than 15 years of age living in the home (yes, no), frequency of religious attendance (once per week, less than once per week, not at all) and region of residence (Eastern Canada (Quebec, Atlantic provinces), central Canada (Ontario) and Western Canada (British Columbia, the Prairies)).

\section{Health status}

Physical health status was assessed by the questions: 'In general, would you say your health is excellent, very good, good, fair or poor?' (excellent/very good/good, fair/poor). 'Are your daily activities at home, work, school or any other area limited by a physical condition?' (sometimes/often/always, no).

Mental health status was assessed by the questions: 'In general, would you say your mental health is excellent, very good, good, fair or poor?' (excellent/very good/good, fair/ poor). 'Are your daily activities at home, work, school or any other area limited by a psychological, emotional or mental health condition?' (sometimes/often/always, no). Three questions asked respondents about their medication use: 'During the past month, have you used any medications that were prescribed or bought over-the-counter to help you 'sleep?' (yes, no), 'calm down?' (yes, no) or 'get out of depression?' (yes, no).

\section{Social support and networks}

Isolation was assessed with the questions: 'How would you describe your sense of belonging to your local community?' (very/somewhat strong, very/somewhat weak). 'Of those relatives and close friends you feel at ease with, how many live in the same city or local community as you?' (none, one or more). 'Do you know of any ethnic or cultural associations or clubs in or near your city or town/community?' and, if so, 'In the past 12 months, were you a member or participant in any of these organisations?' (yes, no).

Trust was assessed with the questions: 'Using a scale of 1-5 wherein 1 means 'cannot be trusted at all' and 5 means 'can be trusted a lot', how much do you trust each of the following groups of people: people in your family, people in your neighbourhood, people you work with or go to school with (asked among those who indicated they were employed or in school), and strangers?' Responses were grouped into 1-3 as low trust and 4 and 5 as high trust.

Finally, experiences of discrimination were assessed with the questions: 'In the past 5 years, have you experienced discrimination or been treated unfairly by others in Canada because of 'ethnicity or culture' (yes, no), 'race or colour' (yes, no), 'religion' (yes, no), or 'language' (yes, no)?' Each of these items was examined separately then combined into an 'any discrimination' (one or more types of discrimination) variable. Respondents who reported discrimination based on any of these items, as well as sex, physical appearance, sexual orientation, age, disability or some other reason were asked, 'Was the discrimination you experienced 'in dealing with public hospitals or health care workers?' (yes, no) or 'from a person in authority or from a service provider?' (yes, no).

\section{Consequences of physical and/or sexual IPV}

Respondents who reported physical and/or sexual IPV were asked how these incidents of violence had affected them.

Respondents answered yes or no to each of the following questions that examined the physical consequences of IPV: 'During this incident/any of these incidents were you ever physically injured in any way, for example bruises, cuts, broken bones, etc?' and 'During the past 5 years, did you ever have to take time off from your everyday activities because of what happened to you other than the time you spent in the hospital or health centre and/or at home in bed?'

The psychological consequences of IPV were assessed by the questions: 'At the time of the incidence/these incidences, how did this experience affect you?' Respondents answered yes or no to each of the following: angry, upset/confused/frustrated, fearful, depression/anxiety attacks and/or lowered self-esteem; and 'During the past 5 years, did you ever fear that your life was in danger because of your (former) spouse/ (former) partner's violent or threatening behaviour?'

\section{Analyses}

Analyses were weighted according to Statistics Canada's guidelines to ensure that the findings were representative of the Canadian population as a whole. We examined the sociodemographic characteristics and prevalence of emotional, financial, physical/sexual and any IPV among immigrant and Canadian-born women reporting contact with a current or former partner in the previous 5 years. Among women reporting any IPV, we compared immigrant and Canadian-born women on the severity of IPV experienced, and sociodemographic, health status and social support and network characteristics. Finally, immigrant and Canadian-born women who reported experiencing physical and/or sexual violence were compared with 
respect to the physical and psychological consequences suffered as a result. All analyses were conducted with a $\chi^{2}$ test for categorical variables. A $p$-value of 0.05 was considered statistically significant. For household income, the proportion of missing data was $14 \%$. Therefore, an unknown/not-stated category was included in the analysis of this variable to retain the sample size.

\section{RESULTS}

A total of 10694 women participated in the 2009 GSS, and of these 6900 reported contact with a current or former partner within the previous 5 years. Information on country of birth was available for 6859 of these women, of whom $5695(83 \%)$ indicated Canada to be their country of birth and $1164(17 \%)$ indicated they were born outside of Canada. A total of 1480 women reported having experienced one or more types of IPV; 1262 were Canadian-born and 218 were immigrants.

There were significant differences in sociodemographic characteristics between immigrant and Canadian-born women (table 1). Immigrant women were more likely to be older $(p=0.02)$ and married or in a common-law relationship $(\mathrm{p}<0.0001)$ and to have more than a high school education $(\mathrm{p}=0.001)$, missing information on household income $(\mathrm{p}<0.0001)$ and children aged $<15$ years living in the home $(p=0.001)$. Compared with Canadian-born women, immigrant women reported more frequent religious attendance $(p<0.0001)$ and were more likely to reside in Central Canada $(p<0.0001)$.

With respect to specific types of IPV, immigrant women were less likely than Canadian-born women to report having experienced emotional $(15.3 \%$ vs $18.2 \%$, $\mathrm{p}=0.04$ ) and physical abuse and/or sexual violence (5.1\% vs $6.9 \%, \mathrm{p}=0.04$; table 2$)$. Compared with Canadian-born women, marginally less immigrant women reported having experienced any type of IPV ( $17.5 \%$ vs $20.3 \%, \mathrm{p}=0.06)$. Among those who reported any IPV, immigrant women were marginally less likely than Canadian-born women to report having experienced two or more types of violence $(\mathrm{p}=0.06)$.

The differences in sociodemographic characteristics between immigrant women and Canadian-born women who reported having experienced any type of IPV were similar to those found in the entire sample. Compared with abused Canadian-born women, abused immigrant women were more likely to be married or living in a common-law relationship ( $\mathrm{p}=0.001$ ), have children aged $<15$ years living in the home $(\mathrm{p}=0.03$ ), have missing information on annual household income $(p=0.01)$, report more frequent religious attendance $(\mathrm{p}=0.0001)$ and reside in Central Canada $(\mathrm{p}<0.0001)$.

There were also differences in health status between abused immigrant and Canadian-born women (table 3). Immigrant women were less likely than Canadian-born women to report that their daily activities were limited

Table 1 Weighted analyses of sociodemographic characteristics of immigrant and Canadian-born women reporting contact with a current or former partner in the previous 5 years in the 2009 General Social Survey ( $N=6859)$

\begin{tabular}{|c|c|c|c|c|c|}
\hline & \multicolumn{2}{|l|}{ Immigrant } & \multicolumn{2}{|l|}{ Canadian-born } & \multirow[b]{2}{*}{ p Value } \\
\hline & Weighted (N) & Per cent & Weighted (N) & Per cent & \\
\hline \multicolumn{6}{|l|}{ Age group } \\
\hline $15-34$ & 277 & 18.3 & 1207 & 22.6 & \multirow[t]{3}{*}{0.02} \\
\hline $35-54$ & 728 & 48.1 & 2467 & 46.2 & \\
\hline 55 and older & 509 & 33.6 & 1672 & 31.3 & \\
\hline \multicolumn{6}{|l|}{ Marital status } \\
\hline Married or common-law & 1406 & 92.9 & 4692 & 87.8 & \multirow[t]{2}{*}{$<0.0001$} \\
\hline Widowed, separated, divorced or single & 107 & 7.1 & 653 & 12.2 & \\
\hline \multicolumn{6}{|l|}{ Education } \\
\hline High school or less & 343 & 22.8 & 1519 & 28.5 & \multirow[t]{2}{*}{0.001} \\
\hline More than high school & 1160 & 77.2 & 3803 & 71.5 & \\
\hline \multicolumn{6}{|l|}{ Annual household income } \\
\hline $0-\$ 19999$ & 73 & 4.8 & 231 & 4.3 & \multirow[t]{4}{*}{$<0.0001$} \\
\hline$\$ 20000-\$ 49999$ & 305 & 20.1 & 1064 & 19.9 & \\
\hline$\$ 50000$ or more & 782 & 51.7 & 3219 & 60.2 & \\
\hline Unknown/not stated & 354 & 23.4 & 832 & 15.3 & \\
\hline Children $<15$ living in the home & 613 & 40.5 & 1831 & 34.3 & 0.001 \\
\hline \multicolumn{6}{|l|}{ Religious attendance } \\
\hline Once per week & 487 & 32.7 & 921 & 17.3 & \multirow[t]{3}{*}{$<0.0001$} \\
\hline Less than once per week & 584 & 39.1 & 2190 & 41.2 & \\
\hline Not at all & 421 & 28.2 & 2207 & 41.5 & \\
\hline \multicolumn{6}{|l|}{ Region of residence } \\
\hline Eastern Canada & 215 & 14.2 & 1940 & 36.3 & \multirow[t]{3}{*}{$<0.0001$} \\
\hline Central Canada & 879 & 58.1 & 1728 & 32.3 & \\
\hline Western Canada & 420 & 27.7 & 1677 & 31.4 & \\
\hline
\end{tabular}


Table 2 Weighted analyses of any intimate partner violence (IPV), and severity of IPV reported by immigrant and Canadian-born women who were in contact with a current or former partner in the previous 5 years

\begin{tabular}{|c|c|c|c|c|c|}
\hline & \multicolumn{2}{|l|}{ Immigrant } & \multicolumn{2}{|l|}{ Canadian-born } & \multirow[b]{2}{*}{ p Value } \\
\hline & Weighted (N) & Per cent & Weighted (N) & Per cent & \\
\hline Any IPV & 256 & 17.5 & 1069 & 20.3 & 0.06 \\
\hline \multicolumn{6}{|l|}{ Type of IPV } \\
\hline Emotional & 225 & 15.3 & 961 & 18.2 & 0.04 \\
\hline Financial & 53 & 3.6 & 240 & 4.5 & 0.18 \\
\hline Physical/sexual & 75 & 5.1 & 362 & 6.9 & 0.04 \\
\hline \multicolumn{6}{|l|}{ Severity of IPV* } \\
\hline Experienced 1 type of abuse & 200 & 69.9 & 741 & 62.1 & 0.06 \\
\hline Experienced 2 or more types of abuse & 86 & 30.1 & 453 & 38.0 & \\
\hline
\end{tabular}

by a physical condition sometimes, often or always $(\mathrm{p}=0.01)$. In addition, immigrant women were less likely than Canadian-born women to report any medication use in the past month for sleep problems $(p=0.05)$ and depression $(\mathrm{p}=0.05)$.

Finally, there were differences between abused immigrant and Canadian-born women in characteristics associated with social supports and networks (table 4). Among those who indicated they knew of cultural associations or clubs, more immigrant women indicated being a member of these organisations in the past 12 months $(p=0.02)$. However, immigrant women were less likely than Canadian-born women to indicate a high level of trust towards their neighbours $(p=0.04)$ or people they work or go to school with $(\mathrm{p}=0.02)$. Moreover, they were more likely than Canadian-born women to report having experienced any discrimination in the previous 5 years $(\mathrm{p}<0.0001)$. Specifically, immigrant women reported more discrimination based on ethnicity or culture $(\mathrm{p}<0.0001)$, race or skin colour $(\mathrm{p}=0.0003)$ and language $(\mathrm{p}<0.0001)$.

There were no differences between immigrant and Canadian-born women in the physical and psychological consequences experienced as a result of physical and/or sexual IPV (table 5).

\section{DISCUSSION}

This study shows that a sizeable proportion of immigrant women in Canada have been affected by violence from a current or former intimate partner. Almost $18 \%$ of immigrant women reported having experienced one or more types of IPV with $15 \%$ having reported emotional abuse, $4 \%$ financial abuse and $5 \%$ physical and/or

Table 3 Weighted analysis of health status of immigrant and Canadian-born women reporting any intimate partner violence

\begin{tabular}{|c|c|c|c|c|c|}
\hline & \multicolumn{2}{|l|}{ Immigrant } & \multicolumn{2}{|c|}{ Canadian-born } & \multirow[b]{2}{*}{$\begin{array}{l}\mathrm{p} \\
\text { Value }\end{array}$} \\
\hline & $\begin{array}{l}\text { Weighted } \\
\text { (N) }\end{array}$ & $\begin{array}{l}\text { Per } \\
\text { cent }\end{array}$ & $\begin{array}{l}\text { Weighted } \\
\text { (N) }\end{array}$ & $\begin{array}{l}\text { Per } \\
\text { cent }\end{array}$ & \\
\hline \multicolumn{6}{|l|}{ Physical health status } \\
\hline \multicolumn{6}{|l|}{ Self-rated physical health } \\
\hline Excellent/very good/good & 228 & 79.6 & 986 & 82.7 & 0.33 \\
\hline Fair/poor & 58 & 20.4 & 206 & 17.3 & \\
\hline \multicolumn{6}{|c|}{ Daily activities limited by physical condition } \\
\hline No & 247 & 86.6 & 929 & 77.8 & 0.01 \\
\hline Sometimes, often or always & 38 & 13.4 & 265 & 22.2 & \\
\hline \multicolumn{6}{|l|}{ Psychological health status } \\
\hline \multicolumn{6}{|l|}{ Self-rated mental health } \\
\hline Excellent/very good/good & 257 & 90.0 & 1059 & 88.9 & 0.67 \\
\hline Fair/poor & 28 & 10.0 & 133 & 11.1 & \\
\hline \multicolumn{6}{|c|}{$\begin{array}{l}\text { Daily activities limited by psychological, emotional } \\
\text { or mental condition }\end{array}$} \\
\hline No & 253 & 89.2 & 1063 & 89.1 & 0.97 \\
\hline Sometimes, often or always & 31 & 10.8 & 130 & 10.9 & \\
\hline \multicolumn{6}{|l|}{ Medication use } \\
\hline Sleep & 40 & 14.1 & 246 & 20.6 & 0.05 \\
\hline Anxiety & 36 & 12.8 & 194 & 16.3 & 0.26 \\
\hline Depression & 33 & 11.5 & 209 & 17.6 & 0.05 \\
\hline
\end{tabular}


Table 4 Weighted analysis of social supports and network characteristics of immigrant and Canadian-born women reporting any intimate partner violence

\begin{tabular}{|c|c|c|c|c|}
\hline Immigrant & & Canadian- & & \\
\hline $\begin{array}{l}\text { Weighted } \\
\text { (N) }\end{array}$ & $\begin{array}{l}\text { Per } \\
\text { cent }\end{array}$ & $\begin{array}{l}\text { Weighted } \\
\text { (N) }\end{array}$ & $\begin{array}{l}\text { Per } \\
\text { cent }\end{array}$ & p Value \\
\hline
\end{tabular}

Isolation

Relatives/close friends at ease with live in same city/local

community

None

One or more

Sense of belonging in your local community

Very/somewhat strong

Very/somewhat weak

Know of ethnic or cultural association/club in/near your city/

town/community

Member of cultural association/club in past 12 months

Trust

Trust family

Low

High

19

Trust neighbours

Low

High

Trust people at work/school

Low

High

34

238

196

79

127

38

262

139

135

Trust strangers

Low

High

Discrimination

Unfair treatment based on:

Ethnicity or culture

Race or colour

Religion

Language

Any discrimination

Unfair treatment when dealing with public hospitals

or health care workers?*

Yes

No

Unfair treatment from a person in authority or from a service provider?*

Yes

No

$$
\text { No }
$$

*Includes those who indicated they had experienced discrimination base
appearance, sexual orientation, age, disability or some other reason.

sexual violence. Almost one-third $(30 \%)$ of immigrant women experiencing IPV reported having experienced two or more types. These estimates are similar to those reported for immigrant women from Canadian population-based surveys in the past. ${ }^{22} 23$

Our study shows that immigrant women were less likely to report having experienced IPV than non-immigrant women, a finding that is consistent with some previous research. ${ }^{19} 2022$ Specifically, immigrant women were less likely than Canadian-born women to report having experienced emotional abuse and physical and/or sexual violence. It may be possible that factors such as embarrassment, stigma, financial dependence on the perpetrator, fears of deportation, a desire to preserve family harmony and honour and community censure for disclosing violence discouraged immigrant women in the survey from reporting IPV. 101416 29-32 In addition, the lower rates of abuse found for immigrant women may be partially explained by the fact that many of the risk factors previously linked to IPV were less likely to be present among immigrant women in this study, such as younger age,$^{20}{ }^{33-35}$ lower educational attainment ${ }^{36}$ and lack of religious attendance. ${ }^{37}$

Although we found no differences between immigrant and Canadian-born women in the consequences of 
Table 5 Weighted analyses of the consequences of physical and/or sexual intimate partner violence for immigrant and Canadian-born women

\begin{tabular}{|c|c|c|c|c|c|}
\hline & \multicolumn{2}{|l|}{ Immigrant } & \multicolumn{2}{|c|}{ Canadian-born } & \multirow[b]{2}{*}{ p Value } \\
\hline & Weighted (N) & Per cent & Weighted (N) & Per cent & \\
\hline \multicolumn{6}{|l|}{ Physical consequences } \\
\hline Injured & 32 & 37.9 & 173 & 42.8 & 0.51 \\
\hline Took time off from everyday activities & 20 & 24.4 & 112 & 27.8 & 0.59 \\
\hline \multicolumn{6}{|l|}{ Psychological consequences } \\
\hline Angry & 21 & 26.7 & 148 & 38.4 & 0.13 \\
\hline Upset/confused/frustrated & 35 & 43.9 & 150 & 38.9 & 0.52 \\
\hline Fearful & 22 & 27.7 & 109 & 28.2 & 0.94 \\
\hline Depression/anxiety attacks & 16 & 20.5 & 96 & 24.9 & 0.52 \\
\hline Lowered self-esteem & 7 & 9.2 & 63 & 16.2 & 0.23 \\
\hline Feared life in danger & 21 & 25.6 & 139 & 34.4 & 0.19 \\
\hline
\end{tabular}

physical and/or sexual IPV, our study confirms the negative impact that such abuse has on women's physical and psychological well-being. About $40 \%$ of immigrant and Canadian-born women who experienced physical and/ or sexual IPV reported being injured as a result of the abuse and approximately $25 \%$ indicated they had to take time off from their everyday activities as a result of the violence. A sizeable proportion of women also indicated that they had suffered psychologically. Over onequarter of women who experienced physical and/or sexual violence reported that the incidence(s) made them feel angry and fearful that their life was in danger.

We found that a number of sociodemographic, health status and social support and network variables differentiated abused immigrant and Canadian-born women. Compared with Canadian-born women, immigrant women were more likely to be married or living in a common-law relationship. Although it is unclear whether immigrant women's current married or common-law partner was the perpetrator of the abuse, factors such as economic dependence or isolation from family and/or friends may discourage immigrant women from leaving an abusive relationship. ${ }^{8} 38$ Immigrant women were also more likely than their Canadian-born counterparts to have young children living in the home, a finding that may be of concern given the well-documented negative impact of children witnessing violence. ${ }^{40-42}$ Having young children in the home may also discourage some immigrant women from leaving the abuser. ${ }^{32}$

Immigrant women were more likely than Canadian-born women experiencing IPV to frequently attend religious services. The research around religious involvement and IPV has been mixed. Data from a national survey of American households showed that regular religious attendance was inversely related to perpetration of IPV. ${ }^{37}$ Some researchers found that personal networks, consisting of religious leaders, family and friends were often the first place abused immigrant women turned to for help. ${ }^{43}$ Others, however, have shown that religious involvement may increase the risk for IPV. It has been speculated that this is owing to the patriarchal ideologies and gender role norms rooted in some religions. ${ }^{14} 45$ In a review of IPV among Korean American immigrant women, Lee and Hadeed ${ }^{14}$ noted that religious affiliation and involvement were significant risk factors for physical assault by a male partner. Religion may also prevent some women from leaving an abusive relationship. ${ }^{32}$ Given these findings, interventions for abuse involving religious communities may be important for immigrant women as they could provide support for those experiencing IPV. Interventions, however, should transform ideologies inherent in some religions that condone or tolerate violence against women.

Generally, abused immigrant women tended to report better physical and mental health than their Canadian-born counterparts. Immigrant women were less likely to report the use of medication in the past month for sleep problems and depression, and to disclose activity limitations due to a physical condition. Greater medication use and activity limitations have both been linked to IPV 333446 and may partially explain the higher rates of abuse among Canadian-born women in this study. For example, previous literature has suggested that women with disabilities may be more vulnerable to abuse because of factors related to limited physical strength and mobility as well as dependence on the abuser for care. ${ }^{47}$

Immigrant women having experienced IPV did not appear to be particularly socially isolated. Similar to Canadian-born women, almost $90 \%$ reported that they had at least one relative or close friend that they felt at ease with in the same city or community and over $70 \%$ felt a strong or somewhat strong sense of belonging in their local community. Moreover, compared with Canadian-born women, a greater proportion indicated that they were a member of an ethnic or cultural association or club near them in the past 12 months. These connections may have contributed in part to immigrant women's assessment of their mental health as being positive despite having experienced IPV. Latta and Goodman $^{44}$ found that family and friends may provide a safe haven for women who experience IPV and a source 
of counselling which may help them cope with the violence.

Immigrant women were more likely than Canadian-born women having experienced IPV to report discrimination in the past 5 years based on culture, ethnicity, race, colour and language. They were also more likely to report lower levels of trust toward their neighbours and the people with whom they work or go to school with. Issues with trust and experiences of discrimination may prevent immigrant women from disclosing the abuse or using social, health and criminal justice services. ${ }^{44} 48$ Previous studies of immigrant women who experienced abuse underscore the need for IPV services that are culturally and linguistically appropriate. ${ }^{10} 32$ In this study, a substantial proportion of women who reported having experienced unfair treatment stated that it occurred when dealing with public hospitals or healthcare workers and from persons in authority or service providers-people that may be integral in women's help-seeking for IPV.

Our exploratory study sheds new light on the physical and psychological consequences of IPV in immigrant and Canadian-born women. Despite this, there are some limitations that need to be kept in mind when interpreting the results. First, while the findings from these analyses suggest that the psychological and physical aftermath of IPV were similar for immigrant compared with Canadian-born women, several different types of consequences for immigrant women could not be reported owing to the small sample size. For consequences that were examined, the sample size may have been too small to detect important differences. Lack of a sufficiently large enough sample also meant that immigrant women had to be grouped into a single category regardless of important factors that may have influenced their experiences of IPV such as the length of residence in Canada. ${ }^{22}{ }^{23}$ The small sample size also inhibited our ability to employ multivariate techniques to determine the relative importance of contextual factors on the consequences of abuse. Future research should use representative samples that are larger and take into account other important factors that may affect immigrant women's experiences of IPV such as the country of origin and age at immigration.

Second, the GSS was administered in English or French only and thus excludes respondents who did not speak either of the official languages. As a result, rates of IPV and associated consequences among immigrant women may have been underestimated, particularly among recent immigrant women who may not have been able to participate in the survey owing to the limited knowledge of English or French. Third, as IPV is a topic that is sensitive in nature, not all women may have acknowledged their experiences of abuse. This may have been particularly true for immigrant women who, as noted earlier, may have had difficulty disclosing IPV owing to particular personal, financial, social and legal concerns. Moreover, although interviewers were trained to detect whether the respondent was alone, many abused women may not have taken the risk of participating in the survey in the first place or disclosing any type of IPV during the interview out of fear of a nearby perpetrator. Fourth, given the cross-sectional nature of this study, it is not possible, for example, to determine whether IPV led to higher rates of activity limitations and/or medication use among Canadian-born women or whether women with activity limitations or higher medication use were more likely to have experienced abuse. Finally, the use of single questions to assess reactions to violence that use labels (ie, depression and anxiety) rather than scales or diagnostic instruments to properly diagnose these conditions may have resulted in the underestimation or overestimation of psychological problems following IPV.

To conclude, this study revealed that a sizeable proportion of immigrant women in Canada have experienced IPV and as a result suffered from a wide range of negative psychological and physical effects. While future research should validate these findings using large, representative samples of diverse groups of immigrant women, these preliminary results suggest that the consequences of IPV are similar for immigrant and Canadian-born women. However, abused immigrant women reported lower levels of trust and were more likely to report being discriminated against for reasons such as race and colour, highlighting some of the structural and systemic factors that may have important implications for seeking help and that underscore the need for IPV-related intervention and prevention services that are culturally sensitive and appropriate.

Contributors Both the authors have contributed equally to this work. JDM conceptualised the study, TF performed the data analysis, JDM and TF together interpreted the results of the data analysis, JDM wrote the main paper and TF wrote the methods section of the paper, JDM and TF jointly reviewed several draughts and edited the paper. Both the authors discussed the results and implications and commented on the manuscript at all stages.

Funding The research for this article was supported by the Public Health Agency of Canada. JDM has been supported by the Atkinson Foundation.

Competing interests None.

Provenance and peer review Not commissioned; externally peer-reviewed.

Data sharing statement No additional data are available.

Correction Notice This paper has been corrected since it was first published. The affiliations have been amended.

\section{REFERENCES}

1. Ansara DL, Hindin MJ. Psychosocial consequences of intimate partner violence for women and men in Canada. $J$ Interpers Violence 2011;26:1628-45.

2. Black MC, Basile KC, Breiding MJ, et al. National intimate partner and sexual violence survey: 2010 summary report. Atlanta, GA: National Center for Injury Prevention and Control, Centers for Disease Control and Prevention, 2011.

3. Carbone-López K, Kruttschnitt C, Macmillan R. Patterns of intimate partner violence and their associations with physical health, psychological distress, and substance use. Public Health Rep 2006;121:382-92 
4. Coker AL, Davis KE, Arias I, et al. Physical and mental health effects of intimate partner violence for men and women. Am J Prev Med 2002;23:260-8.

5. Hathaway JE, Mucci LA, Silverman JG, et al. Health status and health care use of Massachusetts women reporting partner abuse. Am J Prev Med 2000;19:302-7.

6. Romans S, Cohen M, Forte T, et al. Gender and psychotropic medication use: the role of intimate partner violence. Prev Med 2008;46:615-21.

7. Tjaden P, Thoennes N. Extent, nature, and consequences of intimate partner violence:findings from the National Violence Against Women Survey. Washington: Office of Justice Programs, United States Department of Justice, 2000.

8. Alaggia R, Regehr C, Rishchynski G. Intimate partner violence and immigration laws in Canada: how far have we come? Int J Law Psychiatry 2009;32:335-41.

9. Cottrell B. Providing services to immigrant women in Atlantic Canada: Our Diverse Cities. Metropolis Canada, 2008.

10. Guruge S, Humphreys J. Barriers affecting access to and use of formal social supports among abused immigrant women. Can J Nurs Res 2009;41:64-84.

11. Hyman I, Mason R, Guruge S, et al. Perceptions of factors contributing to intimate partner violence among Sri Lankan Tami immigrant women in Canada. Health Care Women Int 2011;32:779-94.

12. Justice Institute of British Colombia. Empowerment of immigrant and refugee women who are victims of violence in their intimate relationships. Vancouver: Child, Family and Community Safety Division Justice Institute of British Columbia, 2007.

13. Kim JY, Sung K. Conjugal violence in Korean American families: a residue of the cultural tradition. J Fam Violence 2000;15:331-45.

14. Lee $\mathrm{Y}$, Hadeed $\mathrm{L}$. Intimate partner violence among Asian immigrant communities: health/mental health consequences, help-seeking behaviors, and service utilization. Trauma, Violence Abuse 2009;10:143-70.

15. Raj A, Silverman J. Violence against immigrant women: the roles of culture, context, and legal immigrant status on intimate partner violence. Violence Against Women 2002;8:367-98.

16. Mason R, Hyman I. Intimate partner violence among immigrant and refugee women. In: Guruge S, Collins E. eds. Intimate partner violence in working with immigrant women: issues and strategies for mental health professionals. Toronto: Centre for Addiction and Mental Health, 2008:279-300.

17. Smith E. Nowhere to turn? Responding to partner violence against immigrant and visible minority women. Ottawa: Canadian Council on Social Development, 2004.

18. Tyyskä V, Mina N. Intimate partner violence against immigrant women: a review of the literature (CERIS Research Grant, 2006-2008). Toronto: Ryerson University, 2005.

19. Hazon A, Soriano FI. Experiences of intimate partner violence among U.S. born, immigrant and migrant Latinas, 2005.https://www. ncjrs.gov/pdffiles1/nij/grants/211509.pdf (accessed 16 Jan 2012).

20. Lown EA, Vega WA. Prevalence and predictors of physical partner abuse among Mexican American women. Am J Public Health 2001;91:441-5.

21. Sorenson SB, Telles CA. Self-reports of spousal violence in a Mexican-American and non-Hispanic white population. Violence Victims 1991;6:3-15.

22. Hyman I, Forte T, Du Mont J, et al. The association between length of stay in Canada and intimate partner violence among immigrant women. Am J Public Health 2006;96:654-9.

23. Ahmad F, Ali M, Stewart DE. Spousal-abuse among Canadian immigrant women. J Immigrat Health 2005;7:239-46.

24. Hurwitz EJ, Gupta J, Liu R, et al. Intimate partner violence associated with poor health outcomes in U.S. South Asian women. $J$ Immigrant Minor Health 2006;8:251-61.

25. Lee J, Pomeroy EC, Bohman TM. Intimate partner violence and psychological health in a sample of asian and caucasian women: the roles of social support and coping. J Fam Violence 2007;22:709-20.
26. Raj A, Silverman JG. Immigrant South Asian women at greater risk for injury from intimate partner violence. Am J Public Health 2003;93:435-7.

27. Statistics Canada. General Social Survey-Victimization (GSS), 2011. http://www23.statcan.gc.ca/imdb/p2SV.pl?

Function=getSurvey\&SDDS=4504\&lang $=e n \& \mathrm{db}=\mathrm{imdb} \& \mathrm{adm}=8 \& \mathrm{dis}=2$ (accessed Sept 2012)

28. World Health Organization. Putting women first: ethical and safety recommendations for research on domestic violence against women. Geneva: World Health Organization, 2001

29. Guruge S, Kanthasamy P, Jokajasa J, et al. Older women speak about abuse and neglect in the post-migration context. Women's Health Urban Life 2010;9:15-41.

30. Mason $\mathrm{R}$, Hyman I, Berman $\mathrm{H}$, et al. 'Violence is an international language': Tamil women's perceptions of intimate partner violence. Violence Against Women 2008;4:1397-412.

31. Hyman I, Mason R, Berman $\mathrm{H}$, et al. Perceptions of and responses to woman abuse among Tamil women in Toronto. Can Womens Stud 2006;25:145-50.

32. Shiu-Thornton S, Senturia K, Sullivan M. 'Like a bird in a cage': Vietnamese women survivors talk about domestic violence. $J$ Interpers Violence 2005;20:959-76.

33. Cohen MM, Forte T, Du Mont J, et al. Intimate partner violence among Canadian women with activity limitations. J Epidemiol Commun Health 2005;59:834-9.

34. Cohen MM, Forte T, Du Mont J, et al. Adding insult to injury: intimate partner violence among women and men reporting activity limitations. Ann Epidemiol 2006;16:644-51.

35. Romans S, Forte T, Cohen MM, et al. Who is most at risk for intimate partner violence? A Canadian population-based study. $J$ Interpers Violence 2007;22:1495-514.

36. Abramsky T, Watts $\mathrm{CH}$, Garcia-Moreno $\mathrm{C}$, et al. What factors are associated with recent intimate partner violence? Findings from the WHO multi-country study on women's health and domestic violence. BMC Public Health 2011;11:109.

37. Ellison C, Anderson K. Religious involvement and domestic violence among U.S. couples. J Sci Study Religion 2001;40:269-86.

38. Amanor-Boadu Y, Messing JT, Stith SM, et al. Immigrant and nonimmigrant women: factors that predict leaving an abusive relationship. Violence Against Women 2012;18:611-33.

39. Erez E, Ammar N, Orloff LE, et al. Violence against immigrant women and systemic responses: an exploratory study. A report submitted to the National Institute of Justice. Washington, DC: US Department of Justice, 2003.

40. Edleson JL. Children's witnessing of adult domestic violence. J Interpers Violence 1999;14:839-70.

41. Holt S, Buckley $\mathrm{H}$, Whelan S. The impact of exposure to domestic violence on children and young people: a review of the literature. Child Abuse Neglect 2008;32:797-810.

42. Wolfe DA, Crooks CV, Lee V, et al. The effects of children's exposure to domestic violence: a meta-analysis and critique. Clin Child Fam Psychol Rev 2003:6:171-87.

43. Bui HN. Help-seeking behavior among abused immigrant women: a case of Vietnamese American women. Violence Against Women 2003:9:207-39.

44. Latta RE, Goodman LA. Considering the interplay of cultural context and service provision in intimate partner violence: the case of Haitian immigrant women. Violence Against Women 2005;11:1441-64

45. Macey M. Religion, male violence, and the control of women: Pakistani Muslim men in Bradford, UK. Gend Dev 1999;7:48-55.

46. Statistics Canada. Family violence in Canada: a statistical profile. Catalogue no. 85-224-X. Ottawa: Statistics Canada, Canadian Centre for Justice Statistics, 2011.

47. Nosek MA, Howland CA, Young ME. Abuse of women with disabilities: policy implications. J Disabil Policy Stud 1997; 8:157-75.

48. Trivedi AN, Ayanian JZ. Perceived discrimination and use of preventive health services. J Gen Interl Med 2006;21:553-8. 


\section{Correction}

Du Mont J, Forte T. An exploratory study on the consequences and contextual factors of intimate partner violence among immigrant and Canadian-born women. BMJ Open 2012;2: e001728. There were errors within the text of this article and affiliation 1 was incorrect. We have since corrected the online version.

BMJ Open 2013;3:e001728corr1. doi:10.1136/bmjopen-2012-001728corr1 